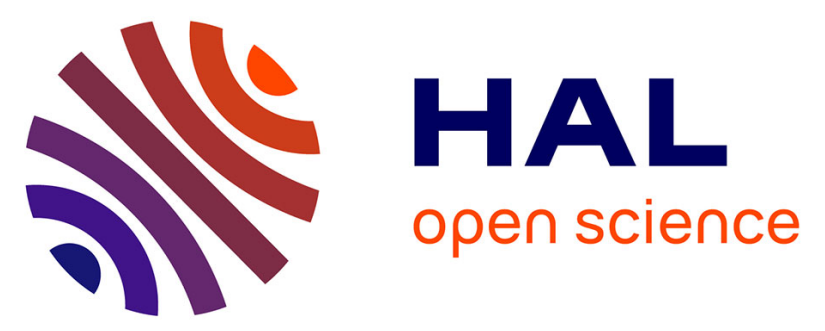

\title{
Analysis of AC Permittivity Response Measured in an Ionic Glass: a Comparison between Iso and Non-iso Thermal Conditions
}

François Henn, Sabine Devautour-Vinot, Jean-Charles Giuntini, Juan Bisquert, Germa Garcia-Belmonte, Christos-Platon E. Varsamis, Efstratios I. Kamitsos

\section{To cite this version:}

François Henn, Sabine Devautour-Vinot, Jean-Charles Giuntini, Juan Bisquert, Germa GarciaBelmonte, et al.. Analysis of AC Permittivity Response Measured in an Ionic Glass: a Comparison between Iso and Non-iso Thermal Conditions. IEEE Transactions on Dielectrics and Electrical Insulation, 2010, 17, pp.1164. 10.1109/TDEI.2010.5539686 . hal-00584851

\section{HAL Id: hal-00584851 https://hal.science/hal-00584851}

Submitted on 11 Apr 2011

HAL is a multi-disciplinary open access archive for the deposit and dissemination of scientific research documents, whether they are published or not. The documents may come from teaching and research institutions in France or abroad, or from public or private research centers.
L'archive ouverte pluridisciplinaire HAL, est destinée au dépôt et à la diffusion de documents scientifiques de niveau recherche, publiés ou non, émanant des établissements d'enseignement et de recherche français ou étrangers, des laboratoires publics ou privés. 


\title{
Analysis of AC Permittivity Response Measured in an Ionic Glass: a Comparison between Iso and Non-iso Thermal Conditions
}

\author{
François Henn, Sabine Devautour-Vinot, Jean-Charles Giuntini \\ Equipe Physicochimie des Matériaux Désordonnés et Poreux, Institut Charles Gerhardt \\ Université Montpellier II, Place Eugène Bataillon, \\ 34095 Montpellier Cedex, France \\ Juan Bisquert, Germà Garcia-Belmonte \\ Photovoltaic and Optoelectronic Devices Group, Departament de Física \\ Universitat Jaume I 12071 Castelló, Spain \\ Cristos Platon E. Varsamis and Efstratios I. Kamitsos \\ Theoretical and Physical Chemistry Institute, \\ National Hellenic Research Foundation, 48 Vass. Constantinou Avenue, \\ 11635 Athens, Greece
}

\begin{abstract}
AC permittivity is measured on a sodium aluminosilicate glass as a function of frequency and temperature using various experimental conditions. The spectra obtained under isothermal and non-isothermal experiments, with insulating barriers placed on both sides of the sample, are compared. An RC equivalent circuit is proposed to account for the experimental behavior and data are analyzed in terms of activation energy distribution. Then, it is pointed out that the dielectric loss signal measured under ramping temperature and fixed frequency conditions lacks of information in the low temperature range. Therefore, the distribution function obtained in that way must be refined in order to account for the high frequency part of the ac permittivity responses measured at fixed temperature and variable frequency. An additional exponential decay contribution to the distribution function appears thus necessary, though its statistical weight is extremely low. This contribution is akin to the so-called CPA (constant phase angle) or NCL (nearly constant loss) behavior.

Index Terms - Ac conductivity, ionic glasses, distribution of activation energy, equivalent $\mathrm{RC}$ circuit
\end{abstract}

\section{INTRODUCTION}

IONIC conduction in condensed matter is one of the primary "machinery" implicated in life, i.e. cationic channels through phospholipidic membrane of biological cells and ionic transport in soils that is the fate of major plant nutrients. It is also involved in many properties of solids, either crystalline or amorphous, for different applications (batteries, fuel cells, sensors, ion exchange membranes, phase separation, catalysis,...) based either on their conductive or dielectric properties [1,2]. Meanwhile, ionic motion in solids and more particularly in disordered ones, i.e. glasses, is not well understood and, so far, no universal model has been acknowledged by the scientific community to account

Manuscript received on 1 September 2009, in final form 28 May 2010. for the broad diversity in experimental behavior [3-5]. At least, the concept of broad distribution of energy barriers for ion hopping initially proposed from models developed in the 70's for amorphous semiconductors [6] and more recently reformulated under the "Random Barrier Model" [7] could be seen as a common point for many solid state ionic glasses. This lack of universal understanding could be due to the fact that both electrical and dielectrical behaviors of these materials were mainly investigated by means of a single method, i.e. complex impedance spectroscopy (CIS) under isothermal conditions, in which dc conductivity and contributions due to polarization effects are not easily separated out. Therefore, it appears that further investigations, based on various experimental conditions and focused on the fundamental aspects of ionic motions in glasses are needed. 
In general, ionic glassy conductors consist of a relatively rigid charged matrix and of ions of opposite charge occupying potential minima. Ions are able to transport the electrical current through the matrix by means of thermally activated hops between different potential minima. Each $j^{\text {th }}$ ionic hop can be characterized by a relaxation time:

$$
\tau_{j}=v_{0, j}{ }^{-1} \exp \left(\frac{E_{j}^{\text {act }}}{k T}\right)
$$

where $v_{0, j}$ is the so-called attempt frequency which ranges in the order of magnitude of atomic vibration, i.e. $10^{13} \mathrm{~s}^{-1}, E_{j}^{\text {act }}$ the activation barrier, $T$ the temperature and $k$ the Boltzmann constant. Depending on the time scale at which the ionic current density is measured, one may observe either (i) long-range redistribution of ions that gives rise to ion diffusion, i.e. dc conductivity, or (ii) local ionic rearrangements causing dipolar reorientation and thus resulting in the intrinsic bulk polarization. It seems, however, that in most cases both longrange and local ionic motions share the same physical origin, i.e. redistribution of ion position above the potential barrier $E^{\text {act }}$ governed by thermally stimulated hopping. This connection can be expressed by the empirical Barton-Nakajama-Namikawa (BNN) proportionality $[8,9]$.

In this paper, we focus exclusively on the dipolar reorientation contribution corresponding to localized hopping motion of the ions. Our aim is to show that a proper analysis of this contribution can provide information, via the determination of $\rho\left(E_{j}^{\text {act }}\right)$, i.e. the distribution of activation energy, about the potential landscape surrounding the ions. In other terms, it is assumed that the knowledge of $\rho\left(E_{j}^{\text {act }}\right)$ is a convenient way to characterize the strength of the ion/matrix interaction and the local structure of the glassy matrix where ions are embedded. The key point is, consequently, to access $\rho\left(E_{j}^{\text {act }}\right)$ from the experimental data. This is not an easy task since, as we have already underlined, electrical measurements using CIS under isothermal experiments and metallic contact between the sample and the spectrometer yield spectra where both dc conductivity and intrinsic polarization contributions overlap. Furthermore, the use of metallic electrode to ensure the electrical contact between the studied sample and the spectrometer is a source of interfacial polarization phenomena which modifies the shape of the spectra. As a consequence, the representation and analysis of dielectric data obtained from CIS has been a controversial subject for decades [3].

In the following, we provide new experimental insights about the intrinsic polarization behavior of ionically conducting glasses by comparing CIS data collected under different experimental conditions, i.e. iso versus non isothermal, using insulating barrier, i.e. PTFE thin film, placed between the metallic current collector of the spectrometer and the sample. The determination of $\rho\left(E_{j}^{\text {act }}\right)$ derives, via an $\mathrm{RC}$ circuit representation, from the resulting expression of the complex ac permittivity $\varepsilon^{\prime \prime}(\omega, T)$ and, finally, the fit of the experimental data. The paper is organized as follows: in Section 2, we briefly recall the main experimental conditions for data acquisition as reported in a previous article [10]. In Section 3, an equivalent $\mathrm{RC}$ circuit is proposed where each branch is chosen based on physical meaningful assumptions. The analysis of the experimental sample responses $\varepsilon^{*}(\omega, T)$ obtained using both iso and non-isothermal conditions are reported in Section 4. Conclusion is given in Section 5 .

\section{EXPERIMENTS}

The sample used in this work is an aluminosilicate ionic glass of chemical formula $\mathrm{Na}_{2} \mathrm{O}-0.4 \mathrm{Al}_{2} \mathrm{O}_{3}-2.2 \mathrm{SiO}_{2}$ obtained by melting mixtures of $\mathrm{Na}_{2} \mathrm{CO}_{3}-10 \mathrm{H}_{2} \mathrm{O}, \mathrm{SiO}_{2}$ and $\mathrm{Al}_{2} \mathrm{O}_{3}$ in platinum crucibles in air at $1500{ }^{\circ} \mathrm{C}$ for one hour and casting into ingots. Samples were annealed at $500{ }^{\circ} \mathrm{C}$ during one hour, and then cooled to room temperature in order to remove internal mechanical stress due to quenching. The so-prepared glassy sample was shaped in a cylindrical form with diameter of 30 $\mathrm{mm}$ and thickness of $2.1 \mathrm{~mm}$. Its parallel faces were optically polished with diamond paste. Ac permittivity measurements in isothermal and non-isothermal conditions were performed by using a Novocontrol Broad Band Dielectric Spectrometer.

The detailed report of the data obtained on this sample using metallic contacts versus insulating barriers and isothermal versus non-isothermal conditions has been published elsewhere [10]. The influences of the heating rate, of the fixed frequency of measurement and of a BIAS were measured and discussed. The main conclusion drawn from the comparison of these data was that the coupling of insulating barrier and of non-isothermal conditions enabled the separation of the different contributions to the electrical signal and, hence, the isolation of the signal due to the intrinsic polarization of the studied sample.

This outcome encourages us to propose a representation of the sample electrical signal by means of an equivalent RC circuit and thus to refine the fitting of the experimental data in order to access $\rho\left(E_{j}^{\text {act }}\right)$ as we will discuss in the following.

\section{CONSTRUCTION OF THE EQUIVALENT RC CIRCUIT}

\subsection{GENERAL CONSIDERATION}

As stated in the introduction, most of ionic solids can be regarded as poor conductors or as leaky dielectrics, meaning that their electrical behavior results from the co-existence of conductive and dielectric properties. This picture is related to the fact that ions can hop (i) above a given threshold activation barrier and then provoke a collective electric charge displacement giving rise to dc conductivity ( $n b$ : this barrier threshold can be seen, by analogy with electronic semiconductor, as an energy gap [11] and/or by analogy with percolation theory as the lowest barrier that connects ionic hops along a conduction path [5,12]) or (ii) independently of 


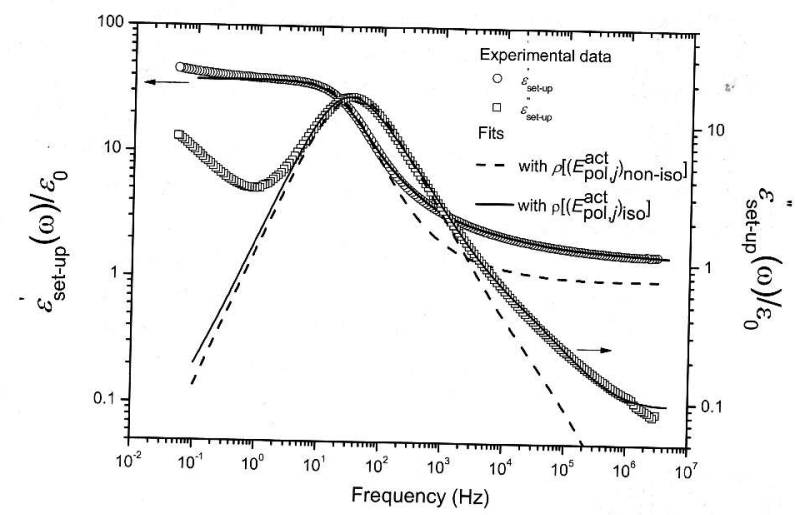

Figure 4. $\log -\log$ plot of the real and imaginary parts of ac permittivity as a function of frequency measured at $313 \mathrm{~K}$. Open circles and squares are the experimental real (left axis) and imaginary (right axis) parts respectively. The dash lines represent the fits using $\rho\left(E_{\mathrm{pol}, j}^{\mathrm{acct}}\right)_{\text {non-iso }}$ as reported in Figure 3; the full lines the fits with the refined distribution function $\rho\left(E_{\mathrm{pol}, j}^{\mathrm{act}}\right)_{\text {iso }}$ as reported in Figure 5. The fit is obtained within the same conditions as for $\rho\left(E_{\mathrm{pol}, j}^{\mathrm{act}}\right)_{\mathrm{non}-\text { iso }}$ but using equation (13) with equation (10) where $\sigma_{\mathrm{dc}}(T)$ follows equation (15) and $\widetilde{v}_{0}$ equals $4.2 \times 10^{12} \mathrm{~s}^{-1}$.

This behavior originates from the corresponding discrepancy between the simulated and experimental non-isothermal response at high temperature values, as already observed in Figure 2, which relates to the space-charge polarization contribution. Second, there is a significant discrepancy both for $\varepsilon_{\text {set-up }}^{\prime}(\omega)$ and $\varepsilon_{\text {set-up }}^{\prime \prime}(\omega)$, in the high frequency domain which corresponds to activation energies lower than that of the peak located at $0.64 \mathrm{eV}$ in Figure 3, i.e. the dc conductivity activation energy. On these grounds, the dielectric response measured at variable frequency and constant temperature is not strictly comparable to that obtained at fixed frequency and ramping temperature in the whole frequency range. This finding suggests that either the first dielectric response misses a part of the intrinsic signal or the second one adds an artifact. Despite the origin of this discrepancy, it is necessary to refine the distribution of $\rho\left(E_{\mathrm{pot}, j}^{\mathrm{act}}\right)$ in order to obtain a satisfactory description of $\varepsilon_{\text {set-up }}^{*}(\omega)$ spectra.

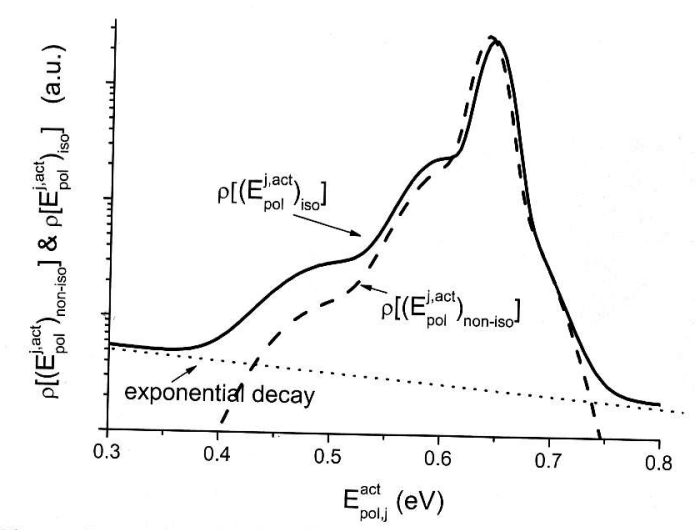

Figure 5. The thick full line is the distribution function of activation energies obtained from the fit of the experimental data reported in Figure 4; the dash line is the distribution function reported in Figure 2. The dot line represents the exponential decay function that must be added to the three Gaussian functions (see Figure 3).
The refined distribution function $\rho\left(E_{\mathrm{po}, j}^{\mathrm{act}}\right)_{\mathrm{iso}}$ that enables us the recovery of the experimental $\varepsilon_{\text {set-up }}^{*}(\omega)$ spectra in the whole explored frequency range is reported in Figure 5. The

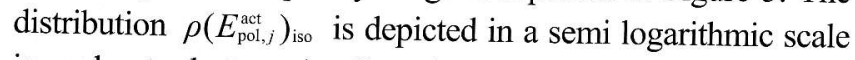
in order to better visualize the weak profile changes. As shown in Figure 5, the distributions $\rho\left(E_{\mathrm{pol}, ~}^{\mathrm{act}}\right)_{\mathrm{iso}}$ and $\rho\left(E_{\mathrm{pol}, j}^{\mathrm{a}, j}\right)_{\mathrm{non}-\text { iso }}$ are rather similar and differ only in an exponential decay contribution of extremely low weight, i.e. about two or three orders of magnitude lower than the others, that is added to $\rho\left(E_{\mathrm{pol}, j}^{\mathrm{act}}\right)_{\mathrm{iso}}$. Although the weight of the additional exponential decay contribution is weak it is mandatory to recover the high frequency response. As reported in Figure 4, at high frequency dielectric loss spectra seem to approach a rather frequency independent regime. This behavior, encountered in ionic solids [5] is often described by the so-called CPE or NCL elements. While there are different possible origins for this behavior, in our case a plausible explanation would be the low energy ion hopping motion around their equilibrium position, in agreement with the assumptions made for the construction of the equivalent circuit (see Figure 1a). It must be noted however that NCL may also originate from interfacial polarization due to capacitances related to the roughness of the contact between the metallic current collector, the PTFE thin layer and the studied sample [16, 17]. However, in this case NCL is not an intrinsic dielectric property of the sample anymore. More detailed investigations on the exact role of the interface should be carried out to clarify this point.

\section{CONCLUSION}

In the present work, we show that the ac permittivity behavior of an ionic conducting glass, i.e. $\mathrm{Na}_{2} \mathrm{O}-0.4 \mathrm{Al}_{2} \mathrm{O}_{3}-2.2 \mathrm{SiO}_{2}$, can be sketched properly by an equivalent circuit made of $\mathrm{R}$ and $\mathrm{C}$ elements mounted in parallel and in series. The idea behind this representation is that ac permittivity results from the superposition of conductive and dielectric contribution. The measure of $\varepsilon_{\text {set-up }}^{*}(\omega)$ in non-isothermal conditions, i.e. linear ramping temperature and fixed frequency, allows us a relatively easy separation of these two contributions. Therefore, the analysis of the dielectric response is feasible and, consequently, the fitting of the experimental spectra with the complex permittivity given by the equivalent $\mathrm{RC}$ circuit yields the distribution function of activation energy, $\rho\left(E_{\mathrm{pol}, j}^{\text {act }}\right)_{\text {non-so }}$, for ion hopping. However, the use of $\rho\left(E_{\mathrm{pol}, j}^{\mathrm{act}}\right)_{\mathrm{non} \text {-iso }}$ for the simulation of $\varepsilon_{\text {set-up }}^{*}(\omega)$ measured under isothermal conditions results in a rather poor description of both the real and imaginary parts of the experimental spectra. In order to reproduce satisfactorily the measured profiles, the refinement of $\rho\left(E_{\mathrm{pol}, j}^{\mathrm{act}}\right)_{\mathrm{non-iso}}$ with the addition of an exponential decay is 
each other between neighbor energy minima localized around a given structural site.

This co-existence can be modeled by an equivalent $\mathrm{RC}$ circuit providing that a $\mathrm{RC}$ parallel element accounts for conductive properties, i.e. conduction relaxation [3], featured by a peak in the $Z_{\text {ac }}^{\prime \prime}(\omega)=f(\omega)$ spectrum whereas an $\mathrm{RC}$ series one sketches the dielectric relaxation properties characterized by a peak in the $\varepsilon_{\mathrm{ac}}^{\prime \prime}(\omega)=f(\omega)$ spectrum. The peculiarity of ionic solids is that resistors and capacitors of both parallel and series elements must be, to some extent, linked to each other in order to account for the BNN relation. Noteworthy, efforts to model complex impedance response of ionic conductors with equivalent circuit have already been made [4] though, most of the time, elements with ambiguous physical meaning are included. For instance, this is the case of the so-called Constant Phase Element (CPE) which is included in the circuit to account for the high frequency part of the polarization conductivity $\sigma_{\text {pol }}^{\prime}(\omega) \propto \omega^{s}$ where $s=1$. Noteworthy, CPE is equivalent to the so-named NCL (nearly constant loss) behavior by others [5].

Here, we propose a simple equivalent circuit (Figure 1) built only from $\mathrm{R}$ and $\mathrm{C}$ elements that can be readily connected to the microscopic phenomena. By "simple", we mean that it can be easily simulated and that a fit of the experimental data is feasible without an excessive number of fitting parameters.

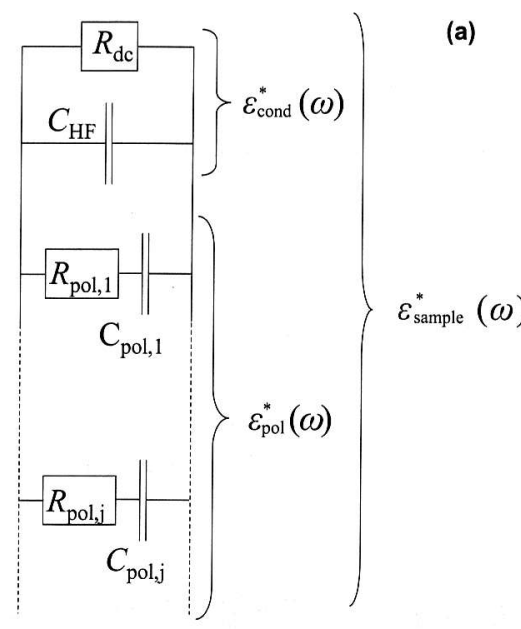

(b)

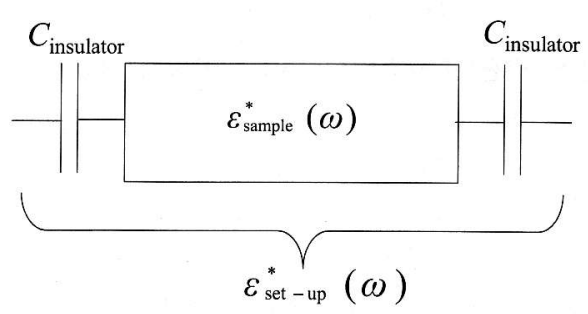

Figure 1. RC circuit model for the intrinsic electrical response of the material (a) and for the set-up when the material is sandwiched between two insulating barriers (b). See the text (section 3) for detailed information about the significance of each component. De conductivity, i.e. $R_{\mathrm{dc}}$ and high frequency permittivity, i.e. $C_{\mathrm{HF}}$ give rise to conductivity relaxation whilst the series components $R_{\mathrm{pol}, j}$ and $C_{\mathrm{pol}, j}$ correspond to the dielectric relaxation.

\subsection{THE EQUIVALENT RC CIRCUIT REPRESENTING THE SAMPLE INTRINSIC RESPONSE}

DC conductivity, $\sigma_{\mathrm{dc}}$, is represented by a resistor, $R_{\mathrm{dc}}=l_{\text {sample }} / A_{\text {sample }} \sigma_{\mathrm{dc}}$ where $l_{\text {sample }}$ and $A_{\text {sample }}$ are the sample thickness and area respectively. The dielectric constant, $\varepsilon_{\mathrm{HF}}$, that corresponds to the material bulk polarization at high frequencies, i.e. the frequency range at which polarization due to localized ionic hops can be neglected, is associated to a capacitor $C_{\mathrm{HF}}=\varepsilon_{\mathrm{HF}} \varepsilon_{0} l_{\text {sample }} / A_{\text {sample }}$ where $\varepsilon_{0}=8.85 \times 10^{-12}$ i.u. is the vacuum permittivity. In an ionic glass, $\varepsilon_{\mathrm{HF}}$ corresponds to the polarity of the network former and eventually to the fastest re-orientational motion of weakly trapped impurities and/or structural defects. $R_{\mathrm{dc}}$ and $C_{\mathrm{HF}}$ are connected in parallel since they are associated to independent physical processes. The resulting complex responses $Z_{\text {cond }}^{*}(\omega)$ and $\varepsilon_{\text {cond }}^{*}(\omega)$ are given by:

$Z_{\mathrm{cond}}^{*}(\omega)=\frac{1}{\left(R_{\mathrm{dc}}\right)^{-1}-i \omega C_{\mathrm{HF}}}$

and

$\varepsilon_{\mathrm{cond}}^{*}(\omega)=\varepsilon_{\mathrm{HF}}+i \frac{\sigma_{\mathrm{dc}}}{\varepsilon_{0} . \omega}$

The imaginary part of the impedance (equation (2a)) exhibits a so-called conduction relaxation whose maximum occurs at $\omega_{Z_{\max }^{\prime \prime}}=\left(R_{\mathrm{dc}} C_{\mathrm{HF}}\right)^{-1}$.

In parallel to this conduction relaxation, we can now add the dielectric relaxation contribution $\varepsilon_{\text {pol }}^{*}(\omega)$ due to the localized, i.e. re-orientational, ionic motions. Based on the assumption that these localized motions occur independently, the global dielectric relaxation contribution can be modeled by a set of RC series elements mounted in parallel (Figure 1a). Each dielectric relaxor $j$ consists of a resistance $R_{\mathrm{pol}, j}$ and a capacitance $C_{\mathrm{pol}, j}$ and is characterized by a relaxation time $\tau_{\mathrm{pol}, j}=R_{\mathrm{pol}, j} C_{\mathrm{pol}, j}$ for which the thermal activation follows equation (1). From the microscopic viewpoint, $R_{\mathrm{pol}, j}$ symbolizes the probability for the ion/network dipole to overpass the activation barrier $E_{j}^{\text {act }}$ related to its reorientational motion and $C_{\mathrm{pol}, j}$ the corresponding variation of dipolar moment $\Delta \varepsilon_{\mathrm{pol}, j}$. For a given dipole $j, \varepsilon_{\mathrm{pol}, j}^{*}(\omega)$ is given by the Debye relaxation function:

$\varepsilon_{\mathrm{pol}, j}^{*}(\omega)=\varepsilon_{\infty, \mathrm{pol}, j}+\frac{\Delta \varepsilon_{\mathrm{pol}, j}}{1+i \omega \tau_{\mathrm{pol}, j}}$

where $\varepsilon_{\infty, \mathrm{pol}, j}$ is the dielectric constant of the dipole at high frequency.

Then for a given distribution function of relaxation times, $\rho\left(\tau_{\mathrm{pol}, j}\right)$, corresponding to the population of dipoles relaxing in parallel (i.e. RC series circuit in parallel), it follows that:

$\varepsilon_{\mathrm{pol}}^{*}(\omega)=\sum_{j} \rho\left(\tau_{\mathrm{pol}, j}\right)\left[\varepsilon_{\infty, \mathrm{pol}, j, j}+\frac{\Delta \varepsilon_{\mathrm{pol}, j}}{1+i \omega \tau_{\mathrm{pol}, j}}\right]$

If we now assume that:

$\forall j, \varepsilon_{\infty, \mathrm{pol}, j}=1$ and $\Delta \varepsilon_{\mathrm{pol}, j}=c s t e=\Delta \varepsilon_{\mathrm{pol}}$ 
Equation (4) yields:

$\varepsilon_{\mathrm{pol}}^{*}(\omega)=\sum_{j} \rho\left(\tau_{\mathrm{pol}, j}\right)+\Delta \varepsilon_{\mathrm{pol}} \sum_{j} \frac{\rho\left(\tau_{\mathrm{pol}, j}\right)}{1+i \omega \tau_{\mathrm{pol}, j}}$

which, due to normalization, i.e. $\sum_{j} \rho\left(\tau_{\mathrm{pol}, j}\right)=1$, becomes:

$\varepsilon_{\mathrm{pol}}^{*}(\omega)=1+\Delta \varepsilon_{\mathrm{pol}} \sum_{j} \frac{\rho\left(\tau_{\mathrm{pol}, j}\right)}{1+i \omega \tau_{\mathrm{pol}, j}}$

Equation (1) shows that $\rho\left(\tau_{\mathrm{pol}, j}\right)$ can be rewritten in terms of distribution of activation energies and attempt frequencies $\rho\left(E_{\mathrm{pol}, j}^{\mathrm{act}}, \ln v_{0, j}\right)$. It is usually assumed that there are no correlations in the distributions of the activation energies $E_{\mathrm{po}, j}^{\mathrm{aat}}$ and in attempt frequencies $v_{0, j}$, so that $\rho\left(E_{\mathrm{po}, j}^{\mathrm{aat}}, \ln v_{0, j}\right)$ is a product of individual distributions:

$\rho\left(E_{\mathrm{pol}, j}^{\mathrm{act}}, \ln v_{0, j}\right)=\rho\left(E_{\mathrm{pol}, j}^{\mathrm{act}}\right) \rho\left(\ln v_{0, j}\right)$

Furthermore, it is reasonable to assume that the distribution of attempt frequencies, $\rho\left(\ln v_{0, j}\right)$, is a narrow one in the sense that the range of attempt frequencies is finite, i.e. rapidly decays for high and low frequencies around the mean peak value $\widetilde{v}_{0}$. Such an assumption is based on the physical nature of $v_{0}$ that represents the atomic vibrational frequency and its value cannot exceed significantly the typical atomic vibrational frequencies comprised roughly in the $10^{12}-10^{14} \mathrm{~s}^{-1}$ range $[13,14]$. It is thus demonstrated [15] that, in such a case, equation (6) can be simplified to:

$\varepsilon_{\mathrm{pol}}^{*}(\omega)=1+\Delta \varepsilon_{\mathrm{pol}} \sum_{j} \frac{\rho\left(E_{\mathrm{pol}, j}^{\mathrm{act}}\right)}{1+i \omega \tau_{\mathrm{pol}, j}}$

with (see equation (1)):

$\tau_{\mathrm{pol}, j}=\widetilde{v}_{0}^{-1} \exp \left(\frac{E_{\mathrm{pol}, j}^{\mathrm{acc}}}{k T}\right)$

Finally, according to the global structure of the RC model circuit, the complex response of the sample is given by $\varepsilon_{\text {sample }}^{*}(\omega)=\varepsilon_{\text {pol }}^{*}(\omega)+\varepsilon_{\text {cond }}^{*}(\omega)$ and by combining equations (2b), (8) and (9) we obtain for the sample permittivity response:

$\varepsilon_{\mathrm{sample}}^{*}(\omega)=1+\varepsilon_{\mathrm{HF}}+\frac{i \sigma_{\mathrm{dc}}}{\omega \cdot \varepsilon_{0}}+\Delta \varepsilon_{\mathrm{pol}} \sum_{j} \frac{\rho\left(E_{\mathrm{pol}, j}^{\mathrm{act}}\right)}{1+i \omega \tilde{v}_{0}^{-1} \exp \left(\frac{E_{\mathrm{pol}, j}^{\mathrm{act}}}{k T}\right)}(10)$

$Z_{\text {sample }}^{*}(\omega)$ can now be determined from equations (10) and (2b).

It must be stressed at that point that if the distribution of relaxation times is restricted to a distribution of activation energies $\rho\left(E_{\mathrm{pol}, j}^{\mathrm{act}}\right)$ then $\varepsilon_{\mathrm{pol}}^{*}(\omega)$ (equation (8)) and consequently $\varepsilon_{\text {sample }}^{*}(\omega)$ (equation (10)) spectra measured at various constant temperatures cannot strictly be superimposed, bearing in mind that as the distribution $\rho\left(E_{\mathrm{pol}, j}^{\mathrm{act}}\right)$ becomes broader, changes in the signal shape with temperature are more significant. It means that for narrow distribution of activation barriers the shape of the isothermal spectra may appear almost temperature independent as it is often reported in the literature in the case of ionic glasses [5]. The fact that this point is still debated indicates that the construction of permittivity or conductivity master curves is not inevitably a universal feature of the electrical properties of ionic glasses. More likely, it emphasizes that it depends on the width of the distribution of activation energies characterizing the ensemble of ionic hops and, hence, on the local structure which can vary a lot from glass to glass.

\subsection{THE EQUIVALENT RC CIRCUIT REPRESENTING THE SAMPLE AND THE INSULATING BARRIERS RESPONSE}

When insulating barriers are inserted between the metallic plates of the spectrometer and the sample, extremely weak dc current flows through the set-up and, hence, in a first approximation space-charge polarization at the interface can be omitted. The resulting equivalent circuit is schematized in Figurelb where $\varepsilon_{\text {sample }}^{*}(\omega)$ is the intrinsic sample response as represented in Figurela and mathematically expressed by equation (10), and $\mathrm{C}_{\text {insulator }}$ is the capacitance of the insulating barriers.

The addition of a capacitance $\mathrm{C}_{\text {insulator }}$ in series implies that the set-up, i.e. sample+insulating barrier, is given by:

$$
C_{\text {set-up }}^{*}(\omega)=\left[C_{\text {sample }}^{*}(\omega)^{-1}+C_{\text {insulatar }}^{*}(\omega)^{-1}\right]^{-1}
$$

and providing that:

$$
C_{\text {set-up }}^{*}(\omega)=\varepsilon_{0} \varepsilon_{\text {set-up }}^{*}(\omega) A_{\text {sample }} / l
$$

where the set-up thickness $l=l_{\text {sample }}+2 l_{\text {insulator }}$, we finally obtain:

$$
\varepsilon_{\text {set-up }}^{*}(\omega)=l\left[\frac{2 \cdot l_{\text {insulator }}}{\varepsilon_{\text {insulator }}^{*}(\omega)}+\frac{1_{\text {sample }}}{\varepsilon_{\text {sample }}^{*}(\omega)}\right]^{-1}
$$

A suitable insulating barrier should behave as a pure capacitance, i.e. $\varepsilon_{\text {insulator }}^{*}(\omega)=\varepsilon_{\text {insulator }}^{\prime}$. In that respect, thin films of PTFE with $l_{\text {insulator }}=10 \mu \mathrm{m}$ and with dielectric constant $\varepsilon_{\text {issulator }}^{\prime}=2.1$ are employed. Since $l_{\text {insulator }}<<l_{\text {sample }}$, $l \cong 1_{\text {sample }}=2.1 \times 10^{-3} \mathrm{~m}$ (see Section 2$)$.

\section{FIT OF THE DIELECTRIC LOSSES SPECTRA MEASURED UNDER NON-ISOTHERMAL CONDITION}

Assuming that $\Delta \varepsilon_{p o l}$ and $\varepsilon_{\text {insulator }}$ are temperature independent [10], then the non-isothermal complex permittivity $\varepsilon_{\text {set-up }}^{*}\left(T, \omega_{a}\right)$ measured at a given fixed pulsation $\omega_{a}$ can be derived from equation (13) where, in that case, $\varepsilon_{\text {sample }}^{*}\left(T, \omega_{a}\right)$ is (see equation $(10)$ in $\left.[10]\right)$ : 


$$
\begin{aligned}
& \varepsilon_{\text {sample }}^{*}\left(T, \omega_{\mathrm{a}}\right)=1+\varepsilon_{\mathrm{HF}}+\frac{i \sigma_{\mathrm{dc}}(T)}{\omega \varepsilon_{0}}+\ldots \\
& \ldots . \Delta \varepsilon_{\mathrm{pol}} \sum_{j} \rho\left(E_{\mathrm{pol}, j}^{\mathrm{act}}\right)_{\mathrm{non}-\mathrm{iso}} \frac{1}{1+i \omega_{\mathrm{a}} \widetilde{v}_{0}^{-1} \exp \left(\frac{E_{\mathrm{pol}, j}^{\mathrm{act}}}{k T}\right)}
\end{aligned}
$$

The sample de conductivity $\sigma_{\mathrm{dc}}(T)$ follows an Arrhenius law [10]:

$$
\sigma_{\mathrm{dc}}(T)=0.3 \exp \left[\frac{-0.64}{k T}\right] \mathrm{Sm}^{-1}
$$

with $k$ expressed in $\mathrm{eV}$. $\widetilde{v}_{0}$ equals $4.2 \times 10^{12} \mathrm{~s}^{-1}$.

In the following, only the imaginary part of $\varepsilon_{\text {set-up }}^{*}\left(T, \omega_{\mathrm{a}}\right)$ obtained from experiments is fitted using equation (15). The adjustment of the experimental data is performed in two steps. First, $\rho\left(E_{\mathrm{pol}, j}^{\mathrm{act}}\right)_{\text {non-iso }}$ is estimated from the fit of $\varepsilon_{\text {set-up }}^{\text {" }}\left(T, \omega_{\mathrm{a}}\right)$. Then, it is refined from the fit of $\log \left(\varepsilon_{\text {set-up }}^{\prime \prime}\left(T, \omega_{\mathrm{a}}\right)\right)$ which allows us a more accurate determination in the low temperature domain where $\varepsilon_{\text {set-up }}^{\prime \prime}\left(T, \omega_{\mathrm{a}}\right)$ is weaker. The comparison between the fitted and experimental spectra is reported in Figure 2 and shows a reasonable agreement up to about $325 \mathrm{~K}$. The discrepancy observed at higher temperatures is due to the space-charge polarization contribution which, despite the presence of PTFE barriers, appears due to the increase of dc conductivity and the resulting accumulation of ionic charges at the interface.

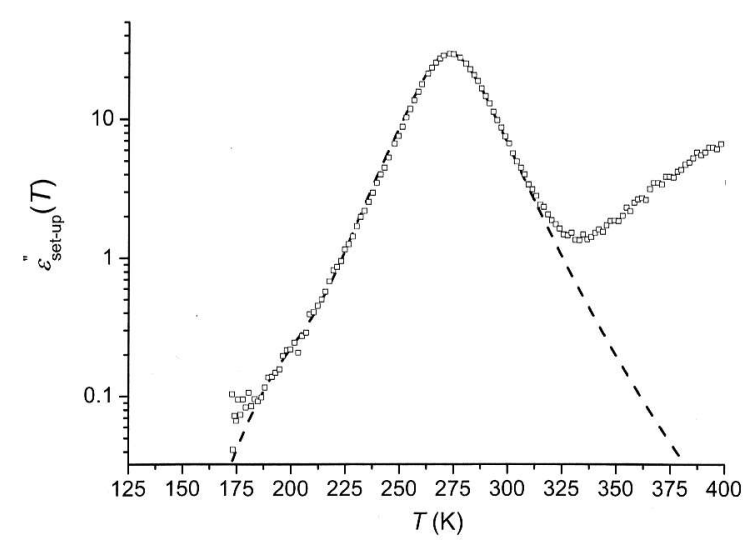

Figure 2. Semi-log plot of the imaginary part of ac permittivity as a function of temperature. Open square are the experimental data obtained on the $\mathrm{Na}_{2} \mathrm{O}-0.4 \mathrm{Al}_{2} \mathrm{O}_{3}-2.2 \mathrm{SiO}_{2}$ sample (see Section 2) and the dash line represents the fit. Experimental data are measured at fixed frequency, i.e. $\omega_{\mathrm{a}}=2 . \pi \mathrm{s}^{-1}$, and linear ramping temperature, i.e. $5 \mathrm{~K} \cdot \mathrm{min}^{-1}$. The fit is obtained using equation (13) with equation (14) where $\sigma_{\mathrm{dc}}(T)$ follows equation (15) and $\tilde{v}_{0}$ equals $4.2 \times 10^{12} \mathrm{~s}^{-1}$. The distribution function $\rho\left(E_{\mathrm{pol}, j}^{\mathrm{ac} t}\right)_{\text {non-iso }}$ extracted from the fit is plotted in Figure 3.

The distribution function of energy, $\rho\left(E_{\mathrm{pol}, j}^{\mathrm{act}}\right)_{\text {non-iso }}$, extracted from the fit (Figure 2) is depicted in Figure 3 and can be decomposed into three Gaussian functions: two dominant components peak located at 0.64 and $0.61 \mathrm{eV}$ and a third one much weaker located at $0.49 \mathrm{eV}$ and not visible in a

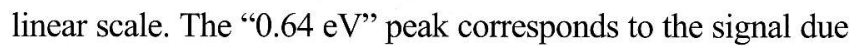
to the PTFE barriers which introduce an additional resonance to the experimental $\varepsilon_{\text {set-up }}^{\prime \prime}\left(T, \omega_{\mathrm{a}}\right)$ spectra. This narrow peak is a single Debye-like relaxation associated to the $C_{\mathrm{PTFE}}-R_{\mathrm{dc}}-C_{\mathrm{PTFE}}$ circuit in series. The two remaining peaks are much broader and correspond to the intrinsic dielectric sample response $\varepsilon_{\text {set-up }}^{\prime \prime}\left(T, \omega_{\mathrm{a}}\right)$.

\section{FIT OF THE COMPLEX PERMITTIVITY SPECTRA MEASURED UNDER ISOTHERMAL CONDITION}

The narrowness of the distribution function reported in Figure 3 implies that the shape of the isothermal spectra should not be significantly temperature dependent. Thus, it is possible to calculate $\varepsilon_{\text {set-up }}^{*}(\omega)$ at any fixed temperature by using as input into equations (10) and (13) the $\rho\left(E_{\mathrm{pol}, j}^{\mathrm{act}}\right)_{\text {non-iso }}$ function obtained under non-isothermal conditions. Following this procedure, we report in Figure 4 the comparison between experimental and simulated $\varepsilon_{\text {set-up }}^{*}(\omega)$ spectra at $\mathrm{T}=313 \mathrm{~K}$. Inspection of Figure 4 shows that by using as input only the distribution $\rho\left(E_{\mathrm{pol}, j}^{\mathrm{act}}\right)_{\text {non-iso }}$, with the rest of parameters fixed as for the fit of $\varepsilon_{\text {set-up }}^{\prime \prime}\left(T, \omega_{\mathrm{a}}\right)$, i.e. $\sigma_{\mathrm{dc}}(T)$ and $v_{0}$, the description of the real and imaginary parts of the experimental spectra is rather poor. First, a significant disagreement between simulated and experimental $\varepsilon_{\text {set-up }}^{*}(\omega)$ spectra is observed in the low frequency domain.

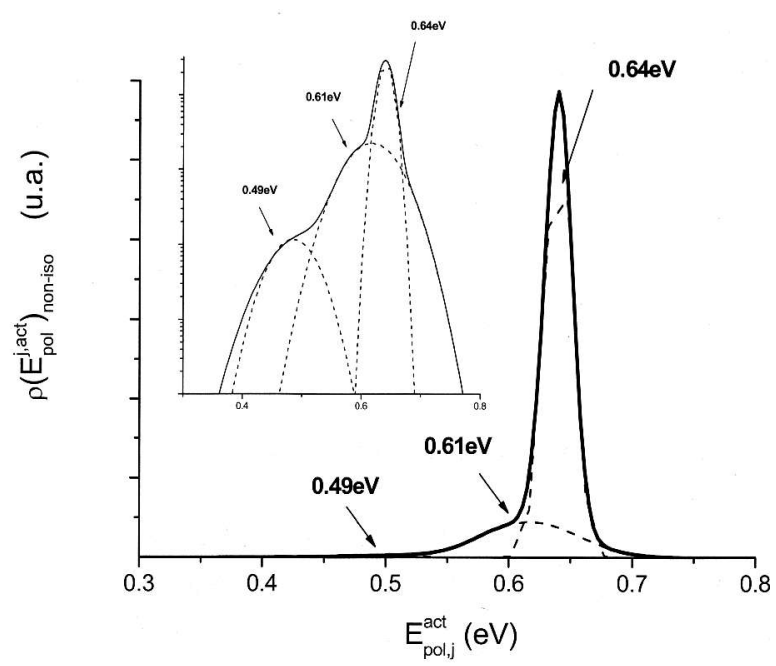

Figure 3. The thick full line is the distribution function of activation energies as a function of activation energies obtained from the fit of the experimental data reported in Figure 2. The dash lines represent the Gaussian functions, the summation of which is the whole distribution function $\rho\left(E_{\mathrm{pol}, j}^{\mathrm{act}}\right)_{\text {non-iso }}$. The inset is the same function plotted in semi-log scale. 
necessary, thus leading to a new distribution $\rho\left(E_{\mathrm{pol}, j}^{\mathrm{act}}\right)_{\text {iso }}$. This extra contribution of low statistical weight corresponds to the so-called CPE or NCL behavior. It is concluded that, in the present case, the additional term relates mostly to the existence of low hopping activation barriers, whose probability decays exponentially with their height. Meanwhile, it should be underlined that different structural features and/or localized defects may be also responsible for such behavior.

\section{ACKNOWLEDGMENT}

The Athens and Montpellier groups are grateful to the Greek and French ministries for Foreign Affairs for their support via the "Platon" program.

\section{REFERENCES}

[1] H. Luo and T.-L. Wen, "The 16th International Conference on Solid State Ionics - Preface “, Solid State Ionics, Vol. 179, p.741, 2008.

[2] See the proceedings of the 2nd International Conference on Physics of Solid State Ionics, Solid State Ionics Vol. 180, pp. 441-662, 2009.

[3] J.R. Macdonald and M.M. Ahmad, "Slopes, nearly constant loss, universality, and hopping rates for dispersive ionic conduction", J. Phys.: Condens. Matter Vol.19, art. 46215, 2007.

[4] J.R. Dygas, "Dielectric function of ionic conductors studied by impedance spectroscopy", Solid State Ionics Vol. 176, pp. 2065-2078, 2005.

[5] J.C. Dyre, P. Maass, B. Roling and D.L. Sidebottom, "Fundamental questions relating to ion conduction in disordered solids“, Rep. Prog. Phys., Vol.72, art. 046501, 2009

[6] F. Henn, S.R. Elliott and J.C. Giuntini, "Complex permittivity in ionically conducting solids: a hopping model", J. Non-Cryst. Sol., Vol. 136, pp. 60-66, 1991.

[7] J.C. Dyre and T.B. Schrøder, "Universality of ac conduction in disordered solids, "Rev. Mod. Phys., Vol.72, pp. 873-892, 2000.

[8] H. Namikawa, "Characterization of diffusion process in oxide glasses based on correlation between electric conduction and dielectric relaxation", J. Non-Cryst. Solids, Vol. 18, pp. 173-195, 1975.

[9] D.L. Sidebottom, B. Roling and K. Funke, "Ionic conduction in solids: Comparing conductivity and modulus representations with regard to scaling properties", Phys. Rev. B, Vol.63, art. 024301, 2001.

[10] F. Henn, G. Garcia-Belmonte, J. Bisquert, S. Devautour-Vinot and J.C. Giuntini, "Dielectric losses measured in a sodium aluminosilicate glass by using electrical insulating barriers and non-isothermal experimental conditions", J. Non-Cryst. Solids, Vol. 354, pp. 3443-3450, 2008.

[11] J.C. Dyre, "Is there a 'native' band gap in ion conducting glasses?", J. Non-Cryst. Solids, Vol. 324, pp. 192-195, 2003.

[12] G. Garcia-Belmonte, F. Henn and J. Bisquert, "Dielectric relaxation strength in ion conducting glasses caused by cluster polarization", Chem. Phys., Vol. 330, pp.113-117, 2006.

[13] E.I. Kamitsos, G.D. Chryssikos, A.P. Patsis and J.A. Duffy, "Metal ion sites in oxide glasses. Relation to glass basicity and ion transport", J. Non-Cryst. Solids, Vol. 196, pp. 249-254, 1996.

[14] G.D. Chryssikos, L. Liu, C.P. Varsamis and E.I. Kamitsos, "Dielectric and structural investigation in alkali triborate glasses", J. Non-Cryst. Solids, Vol. 235-237, pp. 761-765, 1998.

[15] S. Taraskin and F. Henn, "Depolarization current density within the symmetric double-well potential model for solid ionic conductors", Phys. Rev.B, Vol. 77, art. 134306, 2008.

[16] T. Pajkossy, "Impedance spectroscopy at interfaces of metals and aqueous solutions - Surface roughness, CPE and related issues", Solid State Ionics, Vol.176, pp. 1997-2003, 2005.

[17] J.R. Jorcin, M.E. Orazem, N. Pébère and B. Tribollet, "CPE analysis by local electrochemical impedance spectroscopy", Electrochim. Acta, Vol. 51, pp. 1473-1479, 2006.

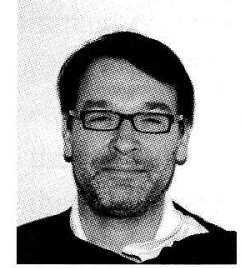

Francois Henn was born in 1964. He received the Ph.D. degree in physical chemistry from the University of Montpellier (France). Then, he was post-doc researcher at Stanford University in the department of Material Sciences and Engineering. He is now full professor of physical chemistry at the University of Montpellier (France) and honorary professor at the "Institut Universitaire de France". He is the leader of a research group associated with CNRS, whose main topics are ionic conductivity and associated dielectric relaxation phenomena in complex systems (e.g. microporous aluminosilicates, oxide glasses, polymers) on the one hand and on the other hand electrochemical behavior of materials for rechargeable batteries (e.g. $\mathrm{Ni}$ electrodes). He can be reached at francois.henn@univ-montp2.fr

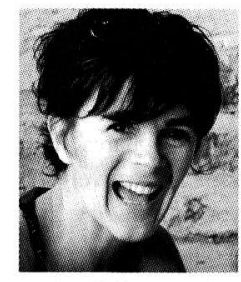

Sabine Devautour-Vinot received her Ph.D. degree in physical chemistry from the University of Montpellier (France), where she is now professor assistant. Her research interest is devoted to the characterization of ionic solids by using dielectric spectroscopy and thermally stimulated current. Her aim is to use these techniques to highlight specific properties of solids as adsorption in porous solids. She can be reached at devaut@lpmc.univ-montp2.fr

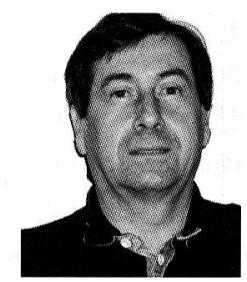

Jean-Charles Giuntini was born in 1945. He received the Ph.D. degree in physical chemistry in 1972 from the University of Bordeaux. During 19721978, he was Assistant Professor at the University of Abidjan (Ivory Cost). He was then appointed Professor at the University of Montpellier II until he retired in 2008. His main research interests were in thermodynamics, adsorption and relaxation phenomena in solids. He can be reached at surjeg@wanadoo.fr

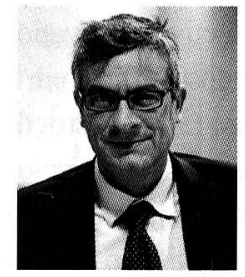

Juan Bisquert is full professor of Applied Physics at Universitat Jaume I de Castelló, where he leads the Group of Photovoltaic and Optoelectronic Devices of 15 physicists and chemists. Recent research activity was focused on nanoscale devices for production and storage of clean energies, in particular dye-sensitized solar cells. Bisquert is specialist in theoretical modeling and interpretation of impedance spectroscopy of electroactive films, transport in disordered materials, interfacial charge-transfer, and the glass transition. He has built up a strong international reputation on the application of measurement techniques and physical modeling that relate the device operation with the elementary steps that take place at the nanoscale dimension: charge transfer, carrier transport, chemical reaction, etc. These methods are currently being applied to dyesensitized solar cells, aligned $\mathrm{ZnO}$ nanowires structures, efficient charge injection and transport in organic LEDs, and solid-state photovoltaic devices. He can be reached at bisquert@fca.uji.es

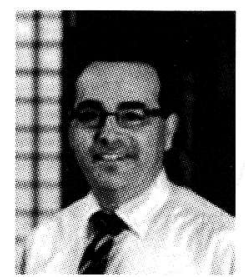

Germa Garcia-Belmonte was born in 1964. He received the Ph.D. degree at Universidad Nacional de Educación a Distancia, in 1996. He worked from 1988 at CIEMAT, Madrid, on experimental as well as theoretical research in the area of digital processing of nuclear signals. He joined the Universitat Jaume I, Castelló, in 1992 and works as Associate Professor of Applied Physics (1999) at the Department of Physics (Photovoltaic and Optoelectronic Devices Group). He investigated the ionic electrical response of glassy and electrochemical systems. Recently he follows research in various topics within the field of organic electronics and photovoltaics as electronic mechanisms in organic light-emitting diodes, organic photovoltaics, and plastic and thin-film solar cells. Device physics using impedance spectroscopy (including modeling and measuring) is his main subject. He can be reached at garciag@fca.uji.es 


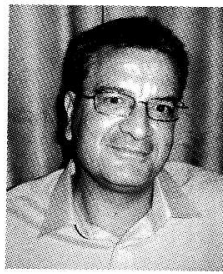

Cristos Platon E. Varsamis has a diploma in physics from the University of Athens (1988), a diploma in physics from the Universita' di Roma La Sapienza (1990), and a Ph.D. degree in solid state physics from the same University in 1994. Since 1999 he is working at the Theoretical and Physical Chemistry Institute of the National Hellenic Research Foundation in Athens. His research interests include the synthesis and structure of ionic glasses by infrared and Raman spectroscopy, their dynamics by impedance spectroscopy and molecular dynamics simulations, and the determination of optical functions of materials by modeling spectroscopic data. He can be reached at cvars@eie.gr

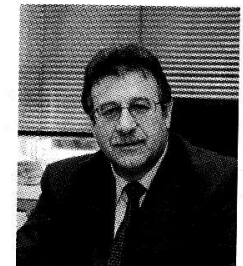

Efstratios I. Kamitsos was born in 1955. He received a diploma in chemistry from the University of Athens in 1978 and the Ph.D. degree in physical inorganic chemistry from Brown University, USA in 1983. In 1985 he joined the Theoretical and Physical Chemistry Institute (TPCI) of the National Hellenic Research Foundation in Athens, and since 1997 he is the Director of the TPCI. His main research interests are in materials physical chemistry with emphasis on structure and dynamics of ionic glasses, and on nanostructured glassy and organic-inorganic hybrid materials with advanced electrical and optical functionality. He can be reached at eikam@eie.gr 